\title{
À PROPOS...
}

\author{
De Boeck Supérieur | «Innovations »
}

$2018 / 1 N^{\circ} 55$ | pages 247 à 257

ISSN 1267-4982

ISBN 9782807391857

Article disponible en ligne à l'adresse :

https://www.cairn.info/revue-innovations-2018-1-page-247.htm

\section{Pour citer cet article :}

«À propos... », Innovations 2018/1 (N55), p. 247-257. DOI 10.3917/inno.055.0247

Distribution électronique Cairn.info pour De Boeck Supérieur.

(C) De Boeck Supérieur. Tous droits réservés pour tous pays.

La reproduction ou représentation de cet article, notamment par photocopie, n'est autorisée que dans les limites des conditions générales d'utilisation du site ou, le cas échéant, des conditions générales de la licence souscrite par votre établissement. Toute autre reproduction ou représentation, en tout ou partie, sous quelque forme et de quelque manière que ce soit, est interdite sauf accord préalable et écrit de l'éditeur, en dehors des cas prévus par la législation en vigueur en France. Il est précisé que son stockage dans une base de données est également interdit. 


\section{À PROPOS...}

\section{Fabienne PICARD, Corinne TANGUY (2017), Innovation et transition techno-écologique, Smart Innovation, Londres, ISTE Editions, 138 p.}

L'ouvrage de Fabienne Picard et de Corinne Tanguy s'intéresse à la question de la transition écologique et énergétique en mobilisant le cadre analytique des systèmes socio-techniques et des systèmes technologiques d'innovation. Il analyse deux grands pans de l'industrie française : l'énergie et l'agriculture/agroalimentaire. Très bien écrit, didactique et complet, le principal mérite de l'ouvrage est de montrer que la transition vers des modèles énergétiques plus durables et agroécologique est fondamentalement un processus. Les auteurs mettent notamment en lumière les nombreux freins, notamment institutionnels, aux changements des modes de production de l'énergie et de l'alimentation. La qualité du raisonnement permet de dépasser une vision techniciste, dans laquelle la transition écologique se traduirait par une seule substitution technologique entre procédés et produits non polluants et nouveaux produits et procédés durables. L'ouvrage se décline en trois parties : la première développe le cadre analytique - principalement issu des transition studies - qui sera confronté aux transitions énergétique et agroécologique, qui font l'objet des deux chapitres suivants.

Le premier chapitre présente l'impasse dans lesquels se trouvent les systèmes socio-techniques contemporains pour faire face aux préoccupations environnementales sans cesse croissantes. Le cadre théorique des transition studies est donc nécessaire pour analyser et saisir le processus de transition vers des modèles de production plus durables. Cet ensemble de cadres analytiques en émergence, comme le précisent à juste titre les deux auteures, se fonde sur deux principes fondamentaux : une approche systémique, qui met en exergue le rôle des modes de coordination entre acteurs et l'architecture institutionnelle du système d'innovation et de production, et le concept de niches, qui sont les moteurs de la transition. Après un examen très complet et didactique de ces différents cadres, la seconde partie du chapitre tend à relativiser l'apport que pourraient avoir les innovations environnementales, technologiques (de produits et procédés) et organisationnelles, liées à la mise en place de nouveaux modèles économiques fondés sur l'écologie industrielle ou sur des logiques d'économie de la fonctionnalité.

Le deuxième chapitre traite de la question de la transition énergétique. Il a le mérite d'exposer le fonctionnement du système énergétique actuel. Les auteurs montrent très justement les limites du modèle énergétique 
actuel - épuisement des ressources énergétiques, dommages environnementaux, émissions de gaz à effets de serre... Elles soulignent également les impasses du maintien des systèmes socio-techniques et des trajectoires technologiques actuelles, dans la recherche d'une amélioration de type incrémental, à travers la séquestration du carbone ou l'amélioration de l'efficacité de l'extraction et de la production des énergies fossiles. Non seulement l'ouvrage montre bien que viser à atteindre la transition énergétique par le biais d'une amélioration des systèmes socio-techniques est une gageure, mais la mise en exergue de la difficulté de la transition vers des modes de production énergétique alternatifs (nucléaire, énergies renouvelables) conduit le lecteur à être pessimiste quant à l'atteinte d'une transition énergétique, pessimisme qui s'accroit encore avec l'illustration de la tentative - vaine - de développer de l'énergie basée sur l'hydrogène...

Le troisième et dernier chapitre s'intéresse à la question de l'agroécologie. Si les enjeux sont différents de ceux du chapitre précédent - incluant notamment les questions liées à l'alimentation et à la santé publique -, les impasses du modèle productiviste français contemporain sont multiples (même s'il est regrettable que la description du modèle actuel n'ai pas fait l'objet d'un développement plus approfondi) : perturbateurs endocriniens, dégradation des sols, des nappes phréatiques et de la biodiversité... Les auteurs présentent ensuite le concept d'agroécologie, sa mise en œuvre et les effets - limités - des innovations environnementales dans l'agriculture et l'agroalimentaire. Les auteurs montrent aussi les limites du développement des circuits courts et de l'agriculture biologique, mais aussi - ce qui distingue ce chapitre du précédent - les apports et la réussite de ces modes de production alternatifs. La transition agroécologique dépendra de la capacité à contourner ou à déverrouiller les multiples blocages présents tout au long de la filière, de l'agriculteur à la production alimentaire.

A la fin de l'ouvrage, le lecteur a le sentiment que si la transition énergétique est un vœu pieu et se heurte à l'état de la science, aux inerties liées aux politiques publiques, aux investissements et aux entreprises établies, la transition agroécologique est en marche : l'agriculture biologique, bon an mal an, se développe de manière continue. De plus, le régime alimentaire d'une partie croissante de la population se transforme, vers une alimentation moins carnée par exemple, même si cet aspect est peu mis en avant dans l'ouvrage. Si le rôle des politiques publiques est moteur pour expliquer la réussite de cette transition, notamment lorsqu'on la compare à la transition énergétique, on remarque le rôle prégnant joué par le consommateur dans cette transition agroécologique. Or, on note qu'un tel rôle du consommateur est absent de l'analyse précédente de la transition énergétique. On pourrait donc s'interroger sur les transformations des préférences, ou sur la plus 
grande prise de conscience de la part du consommateur dans sa consommation énergétique, qui pourraient impulser une véritable transition énergétique. Enfin, le lecteur aurait apprécié une note sur la limite du choix de la mobilisation du cadre théorique des transitions studies, qui, en se concentrant notamment sur le rôle des niches dans la transition, tend à minorer le rôle des consommateurs dans de telles transitions.

En somme, cet ouvrage a le grand mérite de mettre en évidence le caractère processuel lié à toute transition. Son autre grand intérêt est de mettre finement en évidence les freins et blocages institutionnels à la transition, audelà des défaillances de marché et de mécanismes de signal-prix qui appuient la plupart des politiques publiques visant à réorienter les modes de production!

Simon NADEL

Michel BAUWENS, Vasilis KOSTAKIS (2017), Manifeste pour une véritable économie collaborative. Vers une société des communs, Paris, Editions Charles Léopold Mayer, 106 p.

Avec la création et la diffusion du web, une nouvelle phase historique « post-capitaliste » s'est esquissée, au niveau de l'économie mondiale. Cette innovation a démocratisé l'accès aux réseaux, qui atteindrait maintenant presque $40 \%$ de la population mondiale. En promouvant la diffusion de la forme relationnelle " pair à pair » ou P2P, le web permet à des travailleurs et des citoyens, partout dans le monde, de prendre l'initiative de se connecter, mais surtout de s'auto-organiser, de mutualiser des ressources productives. En quoi ces nouvelles formes d'organisation sont-elles effectivement post-capitalistes, que peut-on faire pour les renforcer, et quelle politique du changement peut en découler? Voilà les questions clés auxquelles tentent de répondre ce livre.

Les auteurs du Manifeste pour une véritable économie collaborative défendent l'hypothèse qu' "Internet a créé une occasion historique de reconfigurer la production, l'échange et l'organisation de la société dans son ensemble». Le P2P permet de co-créer des biens communs numériques c'est à dire des savoirs, du logiciel, du design. Ce mode de production est appelée la «production par les pairs basée sur les communs » (PPBC). "C'est le modèle de fonctionnement de l'encyclopédie libre Wikipédia, de la myriade de projets de logiciels gratuits et open source (par exemple Linux, Apache, http serve, Mozilla Firefox) ou de communautés de design ouvert telles que Wikihouse, RepRap et Farmack. » Les auteurs du Manifeste défendent l'hypothèse que la PPBC serait fondamentalement différente des modèles de création de valeur que nous avons connus sous le capitalisme industriel. Dans le cadre de celui-ci, ceux 
qui possèdent le capital financier ou les moyens de production embauchent les travailleurs, dirigent le processus de travail, et vendent les produits dans une recherche de maximisation des profits. Par contre dans la PPBC, fondée sur des systèmes informatiques ouverts et transparents, le travail des participants n'est généralement pas dirigé par des hiérarchies internes à leurs entreprises, mais par des «mécanismes de coordination mutuelle de la communauté productive ». Par exemple le code des logiciels libres et open sources et les entrées Wikipédia sont produits de manière collaborative. Ce nouveau mode de production s'appuie généralement sur trois nouvelles institutions : une communauté productive (ensemble des contributeurs), une coalition entrepreneuriale et une association ou une fondation à bénéfice social (fondation Wikimédia par exemple).

Les auteurs du Manifeste défendent l'idée que «les technologies ne doivent pas être conçues comme neutres, déterministes et univoques quant à leurs effets. A u contraire, nous devrions envisager la technologie comme 'sensible aux valeurs', influencée par les intérêts matériels et les imaginaires sociaux de ceux qui la financent, la développent et l'utilisent. La technologie est un terrain de lutte, où différents intérêts et valeurs s'affrontent ». Ils pensent que « malgré la subordination partielle de l'infrastructure d'Internet aux besoins du capital global, la liberté fondamentale sous-jacente d'Internet n'a pas (encore) été entièrement détruite ». Pour démontrer leur propos les auteurs essaient de schématiser le devenir potentiel du P2P par rapport à deux axes (Centralisé/Localisé et à but lucratif/à bénéfice social), opposant deux modèles génériques de création et de distribution de valeur : d'un côté le modèle « extractiviste » à but lucratif (capitalisme cognitif), et de l'autre le modèle " génératif » à bénéfice social (PPBC mature). Les auteurs proposent dans leur Manifeste une stratégie de transition P2P basée sur les communs et ils soulignent que la production entre pairs ferait ainsi partie des réformes tout à fait essentielles pour rendre l'économie durable sur long terme.

Le Manifeste pour une véritable économie collaborative, qui synthétise de nombreuses publications de la P2Pfondation, est un livre de prospectif dense et stimulant qui montre qu'un autre mode de production de valeur (précisons de valeur ajoutée) est possible, mais qui met trop l'accent sur les dynamiques de transformation internes aux technologies. Il souligne la nécessité d'accompagner cette dynamique technologique par la création de nouvelles institutions, au niveau local, national et global, sans préciser comment ces dernières peuvent se développer dans un contexte politique et culturel peu favorable à leur diffusion. C'est sans doute le point faible de cet ouvrage.

Jacques PERRIN 


\section{Modèle et modes d'organisation des économies africaines}

\section{Abdoul Salam BELLO (2017), La régionalisation en Afrique. Essai sur un processus d'intégration et de développement, Paris, L'Harmattan, $202 \mathrm{p}$.}

Dans une actualité internationale de retour du nationalisme, l'auteur s'intéresse à l'échelle régionale : en quoi est-elle une perspective pour le développement du continent Africain ? Il mobilise, au-delà de son cursus (HEC, Sciences politiques et management), une expérience professionnelle dans le secteur des grandes banques de développement (Banque Mondiale...) et au sein d'institutions régionales Africaines (NEPAD). Il s'appuie sur de la littérature secondaire : médias, rapports, articles académiques.

La première partie du livre rappelle l'histoire politique d'émergence des institutions internationales : les hypothèses, les conditions qui ont présidé au sein des pays industriels à la mise en place d'institutions de régulation de la globalisation et de la structuration d'espaces régionaux comme celui de l'Union européenne. Le terme "régionalisation » y est situé comme étant la création d'espaces sous régionaux transnationaux (par différence avec la notion de région localisée). Ces espaces mutualisent des complémentarités économiques, sociales, voir environnementales entre territoires géographiques au-delà des frontières des Etats-Nations. Cette première partie peu mobilisatrice, constitue un élément de contextualisation institutionnel global de la question posée.

La deuxième partie du livre est centrée sur l'analyse des déterminants historiques et socio-économiques des institutions politiques incarnées par les projets de régionalisation en Afrique. La mobilisation des bases d'informations explique la difficulté d'articuler différentes forces motrices structurantes des réalités institutionnelles qui gouvernent les politiques publiques africaines. La mise en place, l'existence, la gestion des institutions sensées réaliser les opportunités de développement que propose la régionalisation sont questionnées. Cela permet de comprendre la complexité du puzzle institutionnel africain caractérisé par la superposition de deux dynamiques. D'une part, l'émergence de communautés politiques d'Etats: CEMAC, CEA... D'autre part, l'existence d'Unions douanières économiques, parfois monétaires. Dans les deux cas, la gouvernabilité de ces institutions est interrogée au regard de leur faible fonctionnalité. Les accords sur les mécanismes régionaux restent très génériques et orientés par les questions sécuritaires. Les frontières des ensembles régionaux actuels sont pour la plupart ancrées dans les réalités géographique composées par la colonisation. Ces frontières ont parfois déstructuré les processus de régionalisation endogènes aux structures sociales et migratoires historiques qui ont peuplé l'Afrique. 
Les ensembles géographiques constitués par les langues vernaculaires nous en rappellent les contours.

Un élément de difficulté est posé par la permanence du franc CFA (FCFA) qui crée en Afrique une disjonction économique dans la mise en complémentarités et l'intégration économique entre le pays anglophones et francophones. Néanmoins, un processus endogène de structuration de politiques publiques régionales tend à émerger, polarisé par des pays comme le Nigéria, l'Afrique du Sud, le Sénégal et l'Egypte. Le besoin des élites d'affirmation des Etats conduisent cependant à des gouvernances politiques qui privilégient la recherche de légitimités à l'intérieur des frontières nationales. L'existence d'institutions régionales n'apparaitrait dans ce contexte recevable que si elle intervient en complémentarité étroite avec les projets politiques de réalisation des Etats-Nations.

La troisième partie du livre éclaire sur la réalité politique des processus de régionalisation. Elle pose un certain nombre d'hypothèses et permet de caractériser les limites et les opportunités. Elle interroge les perspectives de confirmation des projets en cours. Elle s'appuie en cela principalement sur l'analyse des conditions d'émergence du NEPAD qui structure à la fois un espace politique de convergences des politiques publiques à l'échelle du continent (pris en soit comme une région) notamment en créant les conditions de mise en cohérence d'investissements d'infrastructures (physiques, cognitives) valorisant la mise en complémentarité des territoires. Si l'expérience est politiquement jeune, elle devient une force motrice transnationale qui pourrait dans les années à venir accroitre son rôle comme vecteur d'intégration régionale.

L'auteur identifie plusieurs conditions à ce renforcement. La première est l'adossement à une politique de développement économique plus centrée sur la valorisation des potentialités locales qu'offre le partage de ressources communes (énergétiques, minières, culturelles) pour répondre aux enjeux de la croissance du marché qu'offre la démographie. En cela, la «mise en globalisation » des économies africaines apparait comme une condition de second rang pour le développement économique en rupture avec les recommandations internationales. La deuxième condition mise en débat reste la pertinence du rattachement au FCFA. Au-delà des arguments monétaires de court terme, le maintien d'une situation politique ou les réserves de changes sont sous tutelle de gestion extérieure est une scorie du passé dont il faudra bien un jour s'affranchir.

Pour les lecteurs intéressés par la compréhension des mécanismes de régionalisation et les perspectives de son évaluation en Afrique, ce livre apporte de nouvelles connaissances et des analyses motivantes. Il argumente 
parfois de «points de vue » au sein de différentes controverses mais ces dernières sont en général situées et ces points de vue de se perdent pas dans des positions dogmatiques.

Ludovic TEMPLE

\section{Ahmed SILEM, Cécile BASTIDON, Azzedine GHOUFRANE, Ochozias GBAGUIDI (dir.) (2017), Commerce, investissement et développement durable en Afrique, Le Monde en Question, L'esprit économique, Paris, L'Harmattan, 206 p.}

Cet ouvrage collectif écrit à la suite d'un colloque sur le sujet, réunit une quinzaine d'auteurs (économistes, juristes, sciences politiques) pour analyser en quoi l'évolution des interpellations sur l'environnement et le développement durable impactent le développement du continent africain : des opportunités nouvelles de croissance économique, d'insertion dans le commerce mondial et des contraintes pour le développement. L'ouvrage est organisé en sept chapitres introduits par une synthèse transversale.

Le premier chapitre (P. Hugon) contextualise les enjeux environnementaux liés au changement climatique et la raréfaction des ressources énergétiques mondiales qui interpellent de manière spécifique le développement de l'Afrique. En termes de vulnérabilité, la zone sahélienne est la principale touchée par le réchauffement climatique dans un contexte contradictoire de précarité importante des ressources naturelles et de croissance démographique galopante. En termes de risques environnementaux, l'insertion dans la croissance économique des pays en développement sur le modèle industriel est susceptible de générer une augmentation forte des émissions de carbone à l'échelle mondiale si des politiques environnementales ne sont pas mises en œuvre.

Les chapitres 2 (N. Liouane), 3 (S. Coulibaly) et 4 (M. Ben Abdeljelil S., Ben Ammou) évaluent cette corrélation entre croissance et pollution par des modélisations économétriques sur des données et des indicateurs macroéconomiques respectivement : sur 58 pays en développement, les pays de l'UEMOA et la Tunisie. Ils analysent ainsi la relation entre l'insertion dans le commerce international, la croissance économique et la pollution. Ils tendent à confirmer l'hypothèse d'une courbe en U inversé (Kuznets) selon laquelle la croissance économique nourrie par l'industrialisation augmente la consommation d'énergie et les pollutions en dioxyde de carbone. Cette corrélation peut s'inverser sous certaines conditions de spécialisations économiques. Elles sont précisées dans la possibilité de diversifier les modalités d'insertion des pays en développement dans les échanges internationaux au niveau de la structure de leurs importation et exportations. Cela interpelle 
les politiques de développement et les modèles qui les sous-tendent au regard des risques environnementaux qu'elles font peser sur l'économie mondiale à long terme. Cela souligne l'urgence d'innovations dans les politiques publiques environnementales des pays en développement qui s'industrialisent. La non synchronisation des corrélations entre les pays littoraux et ceux plus sahéliens interpelle les conditions de mise en œuvre de politiques régionales environnementales et énergétiques communes.

Les chapitres 5 (S. Toufik, M. Dinia S. El Baghad, Di S. Nacih), 6 (S. Faucheux, I. Nicolai) et 7 (M. Bekaye) analysent les politiques publiques énergétiques dans les contextes de l'Afrique du nord. Ils montrent en quoi l'Etat volontariste, par une politique d'innovation (législation, soutien financier, formation de capital humain), est l'acteur majeur d'une transition énergétique. Ainsi dans un contexte de croissance de la demande énergétique marocaine la dépendance énergétique extérieure a diminué, mais surtout la démocratisation des conditions d'accès à l'énergie propre liée aux énergies renouvelables est encourageante. La croissance économique et le développement durable sont potentiellement compatibles si leurs interactions sont bien régulées.

Le défi à long terme pour un pays comme le Maroc de promouvoir la croissance verte est potentiellement structuré, en termes de politique d'innovation, par la notion d'éco-innovation sous trois formes. Les innovations dites intégrées (substitution de matières premières), radicales (nouvelles énergies), ou liées à l'économie de la fonctionnalité. Ce défi interroge aussi l'émergence du niveau territorial, de la «ville », comme lieu de mise en cohérence des investissements nécessaires (infrastructures) et de focalisation des opportunités en termes de création/reconversion d'emplois. Les conditions de réussite des projets d'éco-innovation y apparaissent reliées aux capacités de couplage à «l'innovation responsable » dans l'orientation des stratégies d'investissement des entreprises. Curieusement le chapitre 6 conclut sur une condition nécessaire de marchandisation du prix du carbone comme levier d'action prioritaire d'une telle évolution alors que cette conclusion, objet de controverses, a peu été mise en débat ou argumentée en amont.

L'usage de la notion «d'économie verte » en Afrique du nord (Maroc et Tunisie) est testé en mobilisant des indicateurs de suivi qui permettent aux Etats et aux entreprises d'évaluer leur efficacité au regard des objectifs de développement durable. Les auteurs soulignent en quoi les objectifs affichés dans les plans de cadrage des politiques publiques sont difficilement vérifiables en lien avec la fragilité des dispositifs de collecte d'informations environnementales mobilisables.

Ludovic TEMPLE 


\section{Eugène Yves KEDE (2017), La gouvernance climatique au Cameroun, Paris, L'Harmattan, 215 p.}

Cet ouvrage aborde un travail de recherche sur la gouvernance climatique au Cameroun. La problématique du changement climatique étant un enjeu mondial, cet ouvrage présente la situation particulière d'un pays en développement (PED) le Cameroun, sous tous ses aspects liés à la préservation de l'environnement et au développement durable.

L'auteur, Eugène Yves Kede, est docteur en science politique au Cameroun à l'Université de Yaoundé II. Il s'est spécialisé dans les politiques publiques de l'environnement, du développement durable et des questions de sécurité.

A l'entame de son ouvrage, l'auteur présente l'historique de la prise de conscience et de la lutte contre le changement climatique ; considérant que ceci est progressivement devenu un enjeu mondial et global. C'est en 1979 qu'est organisée la première conférence mondiale sur le climat demandant à tous les Etats de la planète de "prévoir et prévenir les conséquences possibles » des changements climatiques. Durant cette conférence a été lancé le programme mondial de recherche sur le climat.

L'auteur met l'accent sur l'analyse du rôle de la Convention Cadre des Nations Unies sur le Changement Climatique (CCNUCC), ainsi que du Groupe d'experts intergouvernemental sur l'évolution du climat (GIEC), les COP à partir de 1994 et le Protocole de Kyoto.

L'ouvrage va davantage s'appesantir sur le Cameroun qui fait partie du Bassin du Congo, second poumon vert de la planète derrière l'Amazonie. Il constitue le deuxième massif forestier mondial. Le Cameroun se situe au cœur des enjeux d'atténuation et d'adaptation au changement climatique constituant un défi pour les politiques publiques. Le changement climatique est devenu, selon l'auteur, une question politique globale et de fait « un objet de négociations internationales pouvant aboutir à des décisions affectant mondialement les pratiques des politiques et économiques ».

Les pays en développement (PED) sont faiblement représentés dans des instances comme le GIEC, et le Cameroun ne fait pas exception. Le Cameroun étant le seul pays du bassin du Congo à avoir un agent de liaison dans le GIEC, il lui est très difficile de défendre les intérêts de tous les pays du bassin du Congo. L'auteur déplore le fait qu'il faut être membre de gros laboratoires de recherche, qui ont des moyens, et qui publient dans les revues scientifiques principales pour pouvoir travailler sur le climat à un niveau international.

L'ouvrage traite principalement du processus d'application du « Reducing Emissions from Deforestation and Forest Degradation» (REDD+) au Cameroun. Le REDD+ étant un instrument de lutte contre le changement 
climatique. Le Cameroun, en tant que membre de la Commission des Forêts de l'Afrique Centrale, porte la question de la déforestation et de la récompense des pays exemplaires en matière de gestion durable et de conservation des couverts forestiers du bassin du Congo. Les forêts du bassin du Congo sont un enjeu avec un intérêt planétaire.

Dans la seconde partie de cet ouvrage, l'auteur est davantage dans une démarche descriptive technique des particularités de la gouvernance climatique au Cameroun. Les multiples acteurs camerounais s'approprient et réinterprètent la matérialisation de l'action publique de lutte contre le changement climatique. On observe des paradoxes dans la mise en œuvre des stratégies d'application du REDD+ sur le terrain. Les ONG internationales et autres organisations gouvernementales, p. ex. le World Wide Fund for Nature (WWF), l'Agence Française de Développement (AFD) et l'Agence allemande de coopération internationale (GIZ), ont la part belle au détriment des ONG locales. Les acteurs gouvernementaux à la manœuvre avec les services ministériels dédiés à la préservation de l'environnement ne se distinguent pas par leur efficacité et leur diligence dans l'application des directives internationales de préservation de l'environnement.

Nous pouvons souligner que les grands pays industrialisés et, de fait, les plus grands pollueurs n'ont pas les mêmes objectifs que les pays en développement. Il apparait que les pays pollueurs veulent influer sur les PED en matière de gouvernance climatique sans que ces derniers ne puissent vraiment faire entendre leur position. La question du financement de la gouvernance climatique est aussi un facteur de déséquilibre. Les pays en développement du bassin du Congo sont en quête de ressources financières et sont en situation de dépendance et d'assujettissement vis-à-vis des grandes puissances. Un pays comme le Cameroun qui a besoin de 28 millions de dollars / an pour appliquer sa politique de gouvernance climatique est en situation de dépendance quasi-totale vis-à-vis des grandes Organisations Non Gouvernementales (ONG) et des grandes institutions internationales des Nations Unies pour l'environnement. Les fonds internationaux pour soutenir la gouvernance climatique mondiale semblent difficiles à lever et prennent des délais très importants pour parvenir aux PED comme le Cameroun.

Sur la forme de l'ouvrage, nous pouvons déplorer le manque de soin : plusieurs oublis de mots rendent certaines phrases incompréhensibles. Le style reprend peut être le jargon technique du domaine des études environnementales et climatiques mais son appropriation est assez laborieuse.

En définitive, cet ouvrage tient autant de l'enquête approfondie sur la gouvernance climatique en Afrique, dans le bassin du Congo et particulièrement au Cameroun que de la recherche scientifique car l'auteur mobilise un 
grand nombre de références scientifiques et analyse dans le détail les mécanismes systémiques des $\mathrm{ONG}$, des ministères, des associations locales et des populations sur le terrain.

Antoine MAHAILET 